\title{
CORRIGENDUM
}

www.nature.com/onc

\section{Pleiotropic regulation of macrophage polarization and tumorigenesis by formyl peptide receptor-2}

\author{
Y Li, L Cai, H Wang, P Wu, W Gu, Y Chen, H Hao, K Tang, P Yi, M Liu, S Miao and D Ye
}

Oncogene (2011) 30, 4373-4374; doi:10.1038/onc.2011.413

Correction to: Oncogene (2011) 30, 3887-3899; doi:10.1038/ onc.2011.112; published online 18 April 2011

The original version of this article contained some errors and mistakes. The corrections are below:

1. In the last paragraph of 'Effects of FPR2 agonists on macrophage polarization' in the 'Results' section:

After the sentence 'To further confirm these results, we then compared the mRNA expression levels of these phenotypes in tumor-infiltrating macrophages (TIMs) from in situ tumor tissue using real-time PCR (Supplementary Figure S2)' add one sentence 'As CXCL8 is not expressed in mouse, we detected another TAM phenotype TGF- $\beta 1$ instead.' and the related reference 'Ma YY, He XJ, Wang HJ, Xia YJ, Wang SL, Ye ZY, Tao HQ et al. (2011). Interaction of coagulation factors and tumor-associated macrophages mediates migration and invasion of gastric cancer. Cancer Sci 102: $336-342$.'

2. The two sentences of the above same paragraph:

'Consistent with the in vitro study, the macrophages in the H22 group showed an M2d-like profile (high levels of IL-10, IL-23p19, IL-6, IL-1 $\beta$, TNF $\alpha$, CCL17, CCL1 and CXCL8; low expressions of IL-12p35 and CXCL13)' and 'IL-23p19, IL-6, IL-1 $\beta$, TNF $\alpha$, CCL1 and CXCL8 were inhibited by ANXA1'

Replace 'CXCL8' with 'TGF- $\beta 1$ '.

3. In the last sentence of 'STAT3 phosphorylation is responsible for FPR2-mediated M2 subset differentiation' in the 'Results' section:

'however, it was not M-CSF + involved in the FPR2 agonist SAA-mediated CCL1 secretion.'

Delete 'M-CSF +'.

4. In Figure 6b, 'CXCL18' should be replaced by 'CXCL8'. The modified Figure 6 is shown below.

5. In the first sentence of the fourth paragraph in 'Discussion' section:

'In many solid tumors, overexpression of M-CSF and MCP correlates'.

'MCP' should be replaced with 'MCP-1'.

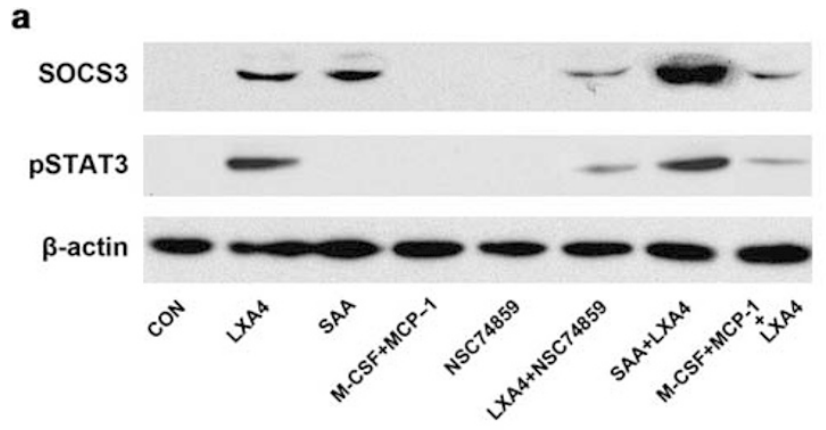

b

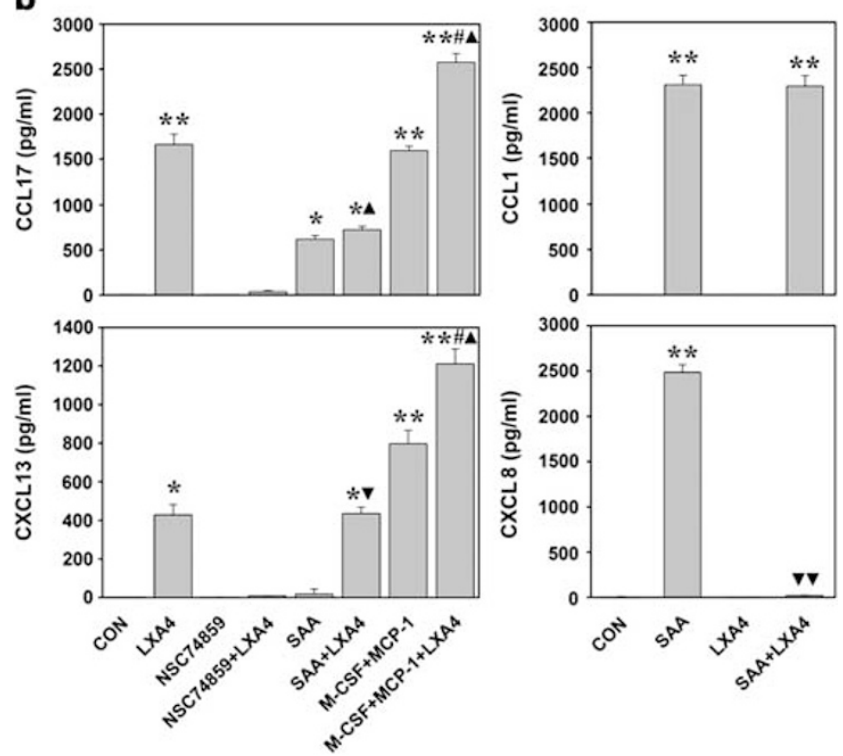

Figure 6 STAT3 signaling is involved in FPR2-mediated macrophage polarization. U937 cells were treated with $\mathrm{LXA}_{4}(100 \mathrm{~nm})$, SAA (100 nM), recombinant human M-CSF $(10 \mathrm{ng} / \mathrm{ml})+$ MCP-1 $(10 \mathrm{ng} / \mathrm{ml})$ and NSC74859 $(100 \mu \mathrm{M})$ for $72 \mathrm{~h}$. (a) The expressions of SOCS3 and pSTAT3 were analyzed by western blotting. (b) The secretions of CCL17, CCL1, CXCL13 and CXCL8 were detected by ELISA. ${ }^{*} P<0.05,{ }^{*} P<0.01$ versus control; ${ }^{\wedge} P<0.05$ versus the $\mathrm{LXA}_{4}$ group; ${ }^{\nabla} P<0.05,{ }^{*} P<0.01$ versus the SAA group; ${ }^{*} P<0.01$ versus the M$\mathrm{CSF}+\mathrm{MCP}-1$ group. CCL, chemokine (C-C motif) ligand; CXCL, chemokine (C-X-C motif) ligand; FPR2, formyl peptide receptor-2; $\mathrm{LXA}_{4}$, lipoxin- $\mathrm{A}_{4} ; \mathrm{MCP}-1$, monocyte chemoattractant protein-1; M$\mathrm{CSF}$, macrophage colony-stimulating factor; SAA, serum amyloid-A; SOCS3, cytokine signaling-3; STAT, signal transducer and activator of transcription; pSTAT, phosphorylated STAT. 
6. In the third sentence of the last paragraph in the 'Discussion' section:

'However, before that, important questions such as a more molecular basis'

Replace 'a more' with 'the exact'.

7. Use 'TGF- $\beta 1$ ' primers in 'Supplementary table' instead of 'CXCL8'
Forward: 5'-GTGTGGAGCAACATGTGGAACTC TA-3'

Reverse: 5'-TTGGTTCAGCCACTGCCGTA-3'

8. In Figure S2C, change 'CXCL8' to 'TGF- $\beta 1$ '. We were previously confused and used the human data of CXCL8. We apologize for that. Mouse does not have CXCL8. We have now provided the modified Figure S2.

\title{
RETRACTION
}

\section{Requirement for chromatin-remodeling complex in novel tumor suppressor HIC1-mediated transcriptional repression and growth control}

\author{
B Zhang, KJ Chambers, D Leprince, DV Faller and S Wang
}

Oncogene (2011) 30, 4374; doi:10.1038/onc.2011.388

Retraction to: Oncogene (2009) 28, 651-661; doi:10.1038/ onc.2008.419; published online 17 November 2008

The authors wish to retract this article after examination of the publication raised concerns that the primary real-time PCR data, which underlie several of the figures in the article, may not be accurate. The integrity of all of the data presented therefore cannot be assured. 